\title{
Numerical simulation of heat transfer augmentation in fin-and-tube heat exchanger with various number of rows of concave rectangular winglet vortex generator
}

\author{
Syaiful $^{1,}{ }^{*}$, ImamSyarifudin ${ }^{2}$, Maria F. Soetanto ${ }^{3}$ and Myung-whanBae ${ }^{4}$ \\ ${ }^{1,2}$ Mechanical Engineering Department, Diponegoro University, Semarang, Indonesia \\ ${ }^{3}$ Aerospace Department, Bandung Polytechnic, Bandung, Indonesia \\ ${ }^{4}$ Department of Mechanical Engineering for Production, Gyeongsang National University, Jinju, \\ South Korea
}

\begin{abstract}
The passive method by using a vortex generator (VG) is an effective method for the improvement of convective heat transfer. This study is focused on usage of concave rectangular winglet vortex generator (CRW VG) for improving convective heat transfer in a fin-and-tube heat exchanger using numerical simulation. Concave rectangular winglet pairs (CRWP) and rectangular winglet pairs (RWP) VGs were mounted inside the gap between fins (gas side) with variations of the number of VG pairs of rows. Inlet air velocity variations expressed by the Reynolds numbers were ranged from 364 to 689 . Augmentation of heat transfer is indicated by the ratio value of heat transfer convection coefficient between cases using VG and that without using VG (baseline). The results show that the convection heat transfer coefficient for cases using CRWP VG is higher than that using RWP VG. Convection heat transfer coefficient increases up to $102 \%$ by mounting CRWP VG at $\mathrm{Re}=364$. However, the increase in convection coefficient is accompanied by a rise in pressure drop to $216.8 \%$.
\end{abstract}

\section{Introduction}

Fin and tube heat exchanger is widely used in industrial, residential and commercial HVACR (heating, ventilation, air conditioning and refrigeration) systems. Various works have been performed to improve the effectiveness of fin and tube heat exchangers because of their impact on the economics of a product. The passive method using VG is one of the most effective methods to improve the effectiveness of the fin and tube heat exchanger by descending the thermal resistance on the gas side [1]. Besides, VG can be used to improve aerodynamic performance of an airfoil [2]. He et al. studied numerically the thermalhydraulic performance of fin-and-tube heat exchanger mounted by rectangular winglet pairs (RWP) VG [3].

*Correspondingauthor: syaiful.undip2011@gmail.com 
Joardar and Jacobi experimentally tested a delta winglet VG mounted on a compact plain fin heat exchanger in a wind tunnel [4]. Investigation on the effect of delta winglet VGs on heat transfer and pressure drop of novel heat exchanger has been studied numerically by Lei et al.[5]. Numerical investigation of the potential of punched winglet type VG arrays for enhancing heat transfer performance was carried out by He et al.[6]. Gholami et al's work applied the wavy-up and wavy-down rectangular winglet VGs for investigating numerically their effect on the heat transfer enhancement and pressure loss penalty in the fin-and-tube compact heat exchangers [7]. Wang et al. examined experimentally the air side performance of fin-and-tube heat exchangers mounting with semi dimple VG [8]. Li et al. evaluated numerically the use of rectangular and delta winglet VGs punched/mounted on fin surfaces for heat transfer augmentation and pressure drop in the fin-and-tube heat exchanger [9]. Wang et al. analyzed the novel longitudinal vortex generator which combined a modified rectangular wing and trapezoidal wing for predicting the performance of finned-tube heat exchanger [10].

Syaiful et al. studied numerically and experimentally the effect of concave delta winglet on the heat transfer improvement in airflow inside a rectangular channel [11]. Numerical investigation of attack angle effect of concave delta winglet vortex generator on the heat transfer augmentation in the fin-and-tube EGR cooler has been carried out by Syaiful et al. [12]. Experimental study of concave rectangular winglet vortex generator effect on the convection heat transfer coefficient of airflow in the rectangular channel has been performed by Syaiful et al. [13]. Therefore, the present work is focused on the effect of various numbers of rows of concave rectangular winglet on heat transfer enhancement in a fin-and-tube heat exchanger using numerical simulation.

\section{Physical and numerical models}

Longitudinal vortex generator (LVG) is modelled by multiple surface protrusions mounted on the fin surface of fin-and-tube evaporator (see Ref. [4]) in the form of RWP and CRWP as denoted in Fig. 1. RWP and CRWP VGs with 1, 3, 4 and 7 rows were mounted in-line with the first, third, fifth and seventh tubes in attack angle of $15^{\circ}$. For CRWP, the radius of the concave surface curvature is $12 \mathrm{~mm}$. VGs in which the high is $60 \%$ of channel high were mounted on the bottom fin surface in the right and left of the tube with the common flow-up orientation.

The numerical model was begun with mesh generation of the model followed by the determination of the boundary condition regions as shown in Fig. 2. Tetrahedral and hexahedral meshes were used for the mesh generation of the model. Fluid flow was assumed as laminar and incompressible with the constant properties. Calculation was performed in the steady state condition. Continuity, momentum and energy equations were applied for solving the physical problems. Conjugate heat transfer was enclosed in the calculation. In order to solve the mathematical equations, boundary conditions were applied to some predetermined regions. Equations of continuity, momentum and energy with some boundary conditions were solved by using computational fluid dynamics code (ANSYS Fluent 14.5). Momentum and energy equations were discretized by using the second order differencing scheme. Pressure and velocity coupling were solved with SIMPLE algorithm. Convergence criteria are revealed by residual in which $10^{-5}$ was for momentum and velocity and $10^{-8}$ was for energy.

Some of the investigated parameters in the present study can be referred to [4] and [14]. Validation is required to obtain accurate prediction results from numerical calculations against the real physical phenomena. In order to reduce the effect of the number of mesh on the change of parameter value, the independent grid was determined by comparing the value of the convection coefficient obtained from the experiment with the value of the nu- 


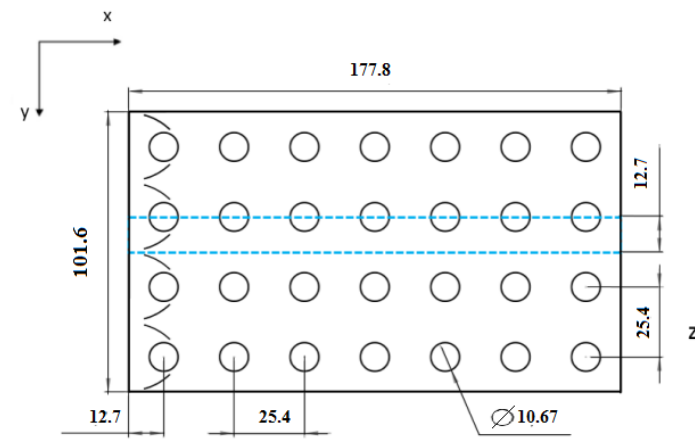

(a)

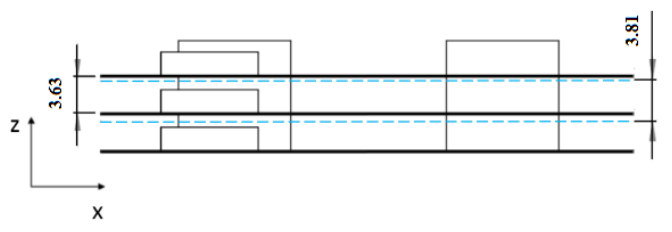

(b)

Fig. 1. Domain computation: a) Top view and b) Side View

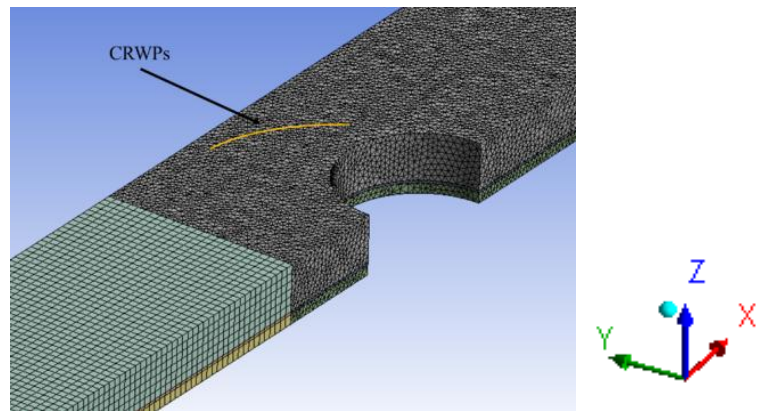

Fig. 2. Mesh generation

merical simulation. Based on the independent grid test, the number of grids was about $1,400,000$ with the smallest deviation of $12.6 \%$. Validation was also done by comparing the results of experiments performed by Joardar and Jacobi [4] with results from the present numerical calculations as expressed in Fig. 3.

As shown in Figure 3, there are similar tendencies for convection coefficient and pressure drop values between the results of these simulations with the results of experiments performed by Joardar and Jacobi [4]. The coefficient of convection and pressure drop increase as the Reynolds number increases for both experiment and

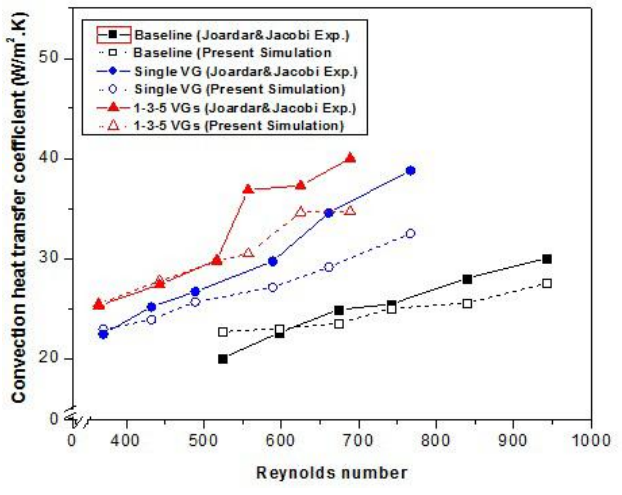

(a) Convection coefficient

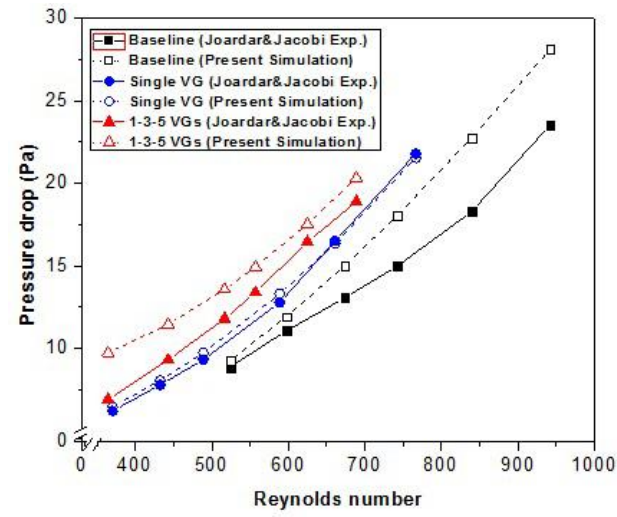

(b) Pressure loss

Fig. 3. Comparison of convection coefficient and pressure drop values between experimental results and present numerical calculations. 
simulation results. By comparing the simulation and Joardar and Jacobi's experimental results, the value of convection coefficients deviate from $8 \%$ to $13 \%$ in the Reynolds number ranges from 523 to 942 . While the pressure drop value deviates from $4 \%$ to $16 \%$ in the same range of Reynolds numbers.

\section{Results and discussion}

Fig. 4 expresses the value of the convection coefficient to the variation of the Reynolds numbers and the number of rows of VG. Baseline in this case means the fins without VG. In general, the coefficient of convection increases with increasing Reynolds numbers for cases with and without VG. Considering the results stated in Fig. 4, the use of RWP and CRWP VG is capable of increasing the convection coefficient compared to the baseline case of the same Reynolds number. This is caused by the ability of VG to produce longitudinal vortex which promotes swirl flow enhancing the flow mixing $[11,12,14]$. This mixing enhances local heat and momentum transfer from the wall to the main flow. These results indicate that the use of CRWP VG improves the convection coefficient better than RWP VG for the same Reynolds number because of stronger longitudinal vortex generated from the CRWP VG than that of RWP VG. Strong longitudinal vortex is caused by instability of flow due to centrifugal force when fluid flows over the concave surface [15, 16]. At $\mathrm{Re}=364$, the use of 1 row of RWP and CRWP VGs increases the value of convection coefficients by $11 \%$ and $22 \%$ respectively to the baseline. The convection coefficient value for case using CRWP VG is doubled than using RWP VG. The highest increase of convection coefficient values are obtained by configuring 7 rows of RWP and CRWP VGs of $38.1 \%$ and $102.5 \%$, respectively. From the results of this simulation it can be concluded that the use of CRWP VG on the fin-and-tube evaporator improves thermal performance better than the use of RWP VG at the same flow rate.

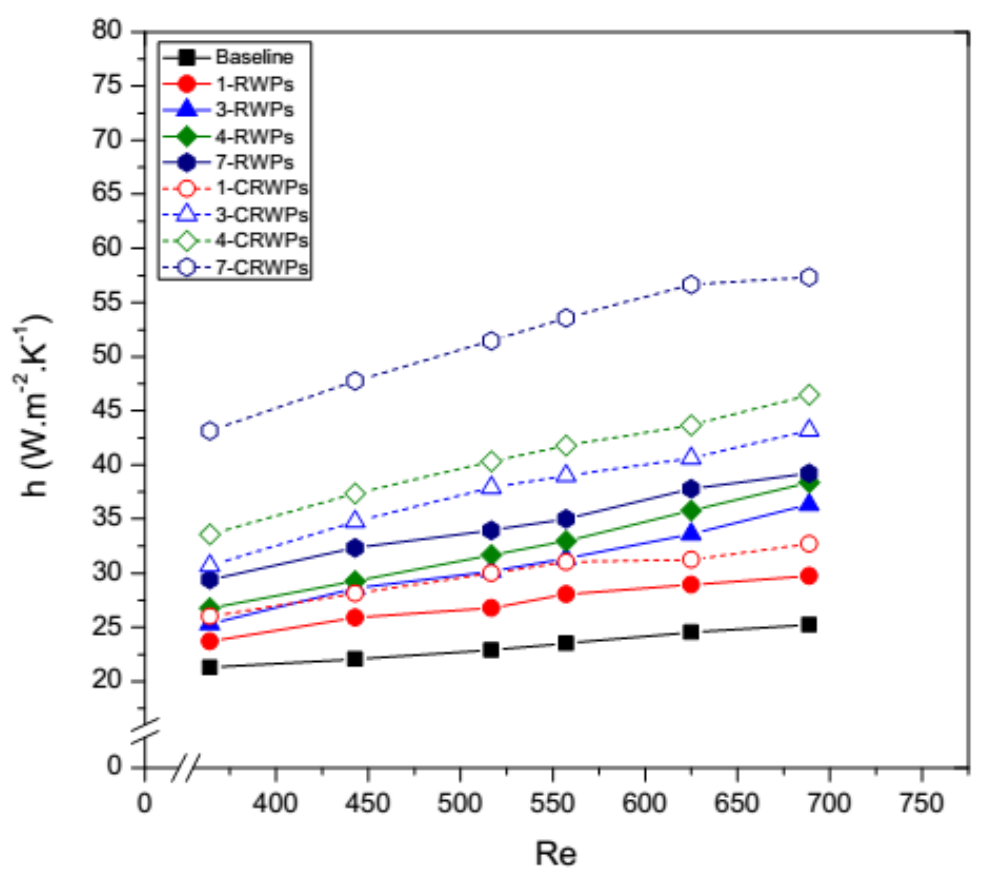

Fig. 4. Comparison of convection coefficient values among baseline, RWP and CRWP VG for variations of Reynolds numbers in difference of number of rows. 
Fig. 5 shows the values of pressure drop with the increase of Reynolds numbers for cases with and without VGs on various number of VG rows. Generally, pressure drop increases with increasing Reynolds number for all cases. This is because the pressure drop is directly proportional to the flow velocity. The lowest pressure drop is observed for the baseline case on all Reynolds numbers due to the absence of a surface protrusion obstructing the flow. The values of pressure drop for the case using CRWP VG are greater than the case using RWP VG for all Reynolds numbers. This is due to the higher size of the projected flow contact field for CRWP VG rather than RWP VG [17]. By comparing to the baseline at $R e=364$, the values of pressure drop in the configuration of 1 row of RWP and

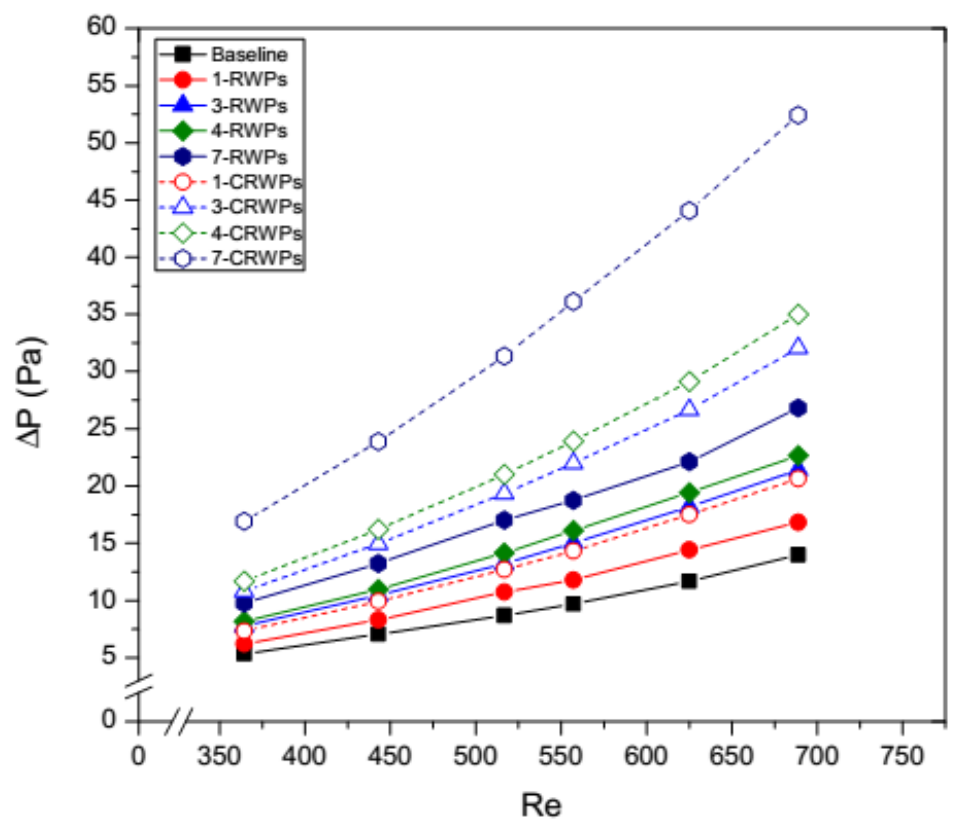

Fig. 5. Comparison of pressure drop values among baseline, RWP and CRWP VG for variations of Reynolds numbers in difference of number of rows

CRWP increase by $16.4 \%$ and $37.1 \%$, respectively. Meanwhile, by mounting 7 rows of RWP and CRWP, the pressure drop values increase to $82.6 \%$ and $216.8 \%$ respectively to the baseline. From the results of this study it can be revealed that the use of CRWP VG in the fin-and-tube evaporator increases flow resistance or decreases of hydrodynamic performance.

\section{Conclusions}

Numerical simulation to determine the effect of concave rectangular vortex generators on the improvement of heat transfer in fin-and-tube heat exchanger has been completed. The calculation validation has been performed by comparing it with the results obtained by the work of Joardar and Jacobi. Agreement between the simulation and the Joardar and Jacobi's experiments results was obtained. In general, the use of concave rectangular winglet pair (CRWP) was more advantageous in terms of thermal performance compared to the use of rectangular winglet pair (RWP). However, the use of CRWP contributed higher increase in pressure drop than that of RWP. 


\section{Acknowledgement}

This work was supported by the Fundamental Research of Indonesian Government (KEMENRISTEK DIKTI, contract number: 699-21/UN7.P4.3/PP/2017), Indonesia. The authors are grateful to all research members, especially Lab. Thermofluid of Mechanical Engineering of Diponegoro University Indonesia, Aerospace Department of Polytechnic of Bandung Indonesia and Advanced Combustion Lab. of Mechanical and Aerospace Engineering Faculty of Gyeongsang National University Korea.

\section{References}

1. B. Delač, AnicaTrp, K. Lenić, "International Journal of Heat and Mass Transfer", 78 (2014)

2. L. Gao, H. Zhang, Y. Liu, S. Han, "Renewable Energy", 76 (2015)

3. Y. L. He, P. Chu, W. Q. Tao, Y. W. Zhang, T. Xie, “Applied Thermal Engineering”, 61 (2013)

4. A. Joardar, A. M. Jacobi, "International Journal of Refrigeration”, 31 (2008)

5. Y. G. Lei, Y. L. He, L. T. Tian, P. Chu, W. Q. Tao, "Chemical Engineering Science”, 65 (2010)

6. Y.L. He, H. Han, W.Q. Tao, Y.W. Zhang, "International Journal of Heat and Mass Transfer", 55 (2012)

7. A.A. Gholami, M. A. Wahid, H.A. Mohammed, "International Communications in Heat and Mass Transfer", 54 (2014)

8. C. C. Wang, Kuan-Yu Chen, Yur-Tsai Lin, “Applied Thermal Engineering”, 88 (2015)

9. Li Li, X. Du, Y. Zhang, L. Yang, Y. Yang, "International Journal of Thermal Sciences", 92 (2015)

10. W. Wang, Y. Bao, Y. Wang, “Applied Thermal Engineering”, 86 (2015)

11. Syaiful, R. Jalil, I. Hambali, M. W. Bae, "Proceedings of the Seventh International Symposium on Mechanics, Aerospace and Informatics Engineering", (2015)

12. Syaiful, G. Reviansyah, M. W. Bae, "FISITA world automotive congress", (2016)

13. Syaiful, G. Sugiri, M. F. Soetanto, M. W. Bae, "American Institute of Physics”, 1788 (2017)

14. Y. L. He, P. Chu, W. Q. Tao, Y. W. Zhang, T. Xie, “Applied Thermal Engineering”, 61 (2013)

15. P. D. McCormack, H. Welker, M. Kelleher, "Journal of Heat Transfer", ASME (1970)

16. V. Malatesta, L. F. Souza, J.T. C. Liu, M. J. Kloker, "Procedia IUTAM", 14 (2015)

17. G. Zhou, Z. Feng, "International Journal of Thermal Sciences”, 78(2014) 